inn that offer offices, for example, pool and web in the meantime. From this inquiry, we could first acquire the whole lodging whose administrations contain the arrangement of decisive words, and after that locate the closest one from the recovered eatery. The real disadvantage of this methodology is that, on the troublesome data they don't give continuous answer. Case in point, from the question point the genuine neighbor lies far away, while all the closer neighbors are lost no less than one of the inquiry magic words. Spatial pivotal word questions have not been broadly investigated. In the previous years, the gathering of individuals has demonstrated enthusiasm for considering pivotal word seek in social databases. As of late the consideration has engrossed to multidimensional information [5][6]. The best system for closest neighbor look with decisive words is a result of Felipe et al. [5]. They join the spatial file R-tree [7] and mark record [8]. So they added to a structure called IR2 - tree. This tree has the capacity of both R-tree and mark records. Like R-tree it stores the spatial vicinity of item and like mark document it channels those articles that do exclude all inquiry pivotal words.

For substance comparability pursuits taking into account highlight vectors, characterizing the separation for speaking to the size of likeness is vital. Euclidean separation is an ordinary measure of separation, yet there are a few issues with this separation as takes after: (1) Euclidean separation is to a great degree delicate to the sizes of the component qualities, and (2) Euclidean separation is incognizant in regards to connected elements. In this paper, we propose a quick multidimensional closest neighbor look calculation in view of ellipsoid separation. Ellipsoid separation considers the connection among elements in ascertaining separation. By utilizing ellipsoid separation, the issues of scale and connection innate in Euclidean separation are no more an issue. With the calculation proposed in this paper, proficient end of pointless number-crunching operations has been accomplished by changing over the computation of ellipsoid separation to figuring of Euclidean separation through a spatial change performed utilizing Cholesky deterioration.

\section{Related Work:}

In the paper 'quick closest neighbor seek with watchwords', there are systems like spatial file, altered list, closest neighbor look. The principal strategy spatial file is utilized for making records in light of the fact that there is immense measure of information should be put away for looking that information put away as xml archives. In the event that the information stockpiling made as records then space needed is less additionally time required for looking the decisive word is less. Second technique is reversed list. The rearranged file information structure is a focal part of a run of the mill web index indexing calculation. An objective of an internet searcher execution is to advance the rate of the question: discover the archives where word happens. When a file is produced, which procurements arrangements of words per archive; it is next rearranged to build up an upset record. Questioning the list would require consecutive cycle through every report and to every word to check a coordinating record. The time memory and preparing property to execute such an inquiry are not generally hypothetically reasonable. As opposed to posting the words per article in the record, the transformed list information structure is created which records the archives per word. The rearranged record delivered, the question can now be dictated by bouncing to the word id in the altered list. These were successfully transformed lists with a little measure of supplementary clarification that obliged an impossible measure of endeavor to deliver. Third strategy is closest neighbor look. Closest neighbor seek (NNS), additionally recognized as closeness inquiry, parallel quest is an advancement issue for discovering nearest focuses in metric spaces. In the paper 'Productive Keyword-Based Search for Top-K Cells in Text Cube' strategies utilized are rearranged record one-sweep, archive sorted-output, base up element programming, and pursuit space requesting. In the top $\mathrm{k}$ cells, there is a seeking of closest key to the question. Blocks structures bunches of single interesting gathering which demonstrates its personality. Strategy like reversed record utilized for giving list as opposed to giving entire information which can be space devouring.

A spatial database oversees multidimensional articles, (for example, focuses, rectangles, and so forth.), and gives quick access to those items taking into account diverse choice criteria. The significance of spatial databases is reflected by the comfort of demonstrating elements of reality in a geometric way [3]. Case in point, areas of eateries, inns, healing centers thus on are regularly spoken to as focuses in a guide, while bigger degrees, for example, stops, lakes, and scenes frequently as a mix of rectangles [4]. Numerous functionalities of a spatial database are valuable in different routes in particular connections [5]. 
III. Nearest neighbor search of multidimensional data:

A nearest neighbor search in a multidimensional space is the problem of finding the nearest vector to a given vector (query vector) $\mathrm{q}$ among $\mathrm{N}$ data vectors (candidate vectors) $x i(i=1,2, \ldots, N)$ placed in $n-$ dimentional space. There are two typical varieties of nearest neighbor search: (i) k-nearest neighbor search (search restricted by number) The search attempts to find the $\mathrm{k}$ vectors closest to the given query vector $\mathrm{q}$, (ii) $\varepsilon$-nearest neighbor search (search restricted by range) The search attempts to find vectors within a distance $\varepsilon$ from the given query vector q; that is, vector xi satisfying $d(q, x i)$ are found. In a linear search wherein a given vector is compared sequentially to all vectors in a database, the computational complexity increases in direct proportion to the database size. Therefore, the development of multidimensional indexing techniques for efficient nearest neighbor search has been attracting much attention recently. 5 There are various algorithms for multidimensional indexing in a Euclidean space, such as R-tree, $6 \mathrm{R}+$ tree, $7 \mathrm{R}^{*}$-tree, $8 \mathrm{SS}$-tree, $9 \mathrm{SS}+$-tree, $10 \mathrm{CSS}+$-tree, $11 \mathrm{X}$ tree, 12 and SR-tree, 13 as well as more general indexing methods for metric spaces, for example, VP-tree,14 MVP-tree,15 M-tree,16 etc. Such indexing techniques are based on restriction of the search range by hierarchical partitioning of multidimensional search space, and they limit the scope of the basic search.

we use the VP-tree as the object of comparison with the proposed method. The following is a brief overview of the VP-tree. The VP-tree is a method for multidimensional indexing of typical distance space. It aims to shrink the amount of space explored in the search by recursively partitioning multidimensional space, based on the distance between data points. The VP-tree uses a reference point known as a vantage point, and it has the special characteristic of not allowing a common area to arise in the partitioned space so that hyperspheres can be used to partition space in a top-down manner. By contrast, the M-tree, which partitions space in a bottom-up manner, has a drawback in that there are many common areas between the partitioned spaces, with the result that search efficiency declines. VP-tree index building can be summarized as follows. A vantage point (hereinafter referred to as $v p$ ) is selected for dataset $\mathrm{s}$ consisting of $\mathrm{N}$ number of data points by means of the random algorithms described below. (i) Select temporary vp randomly from the data set, (ii) Calculate the distance to the reset of the $\mathrm{N}-1$ objects from the temporary, (iii) Calculate the intermediate value and distribution of these distances, (iv) The point of maximum distribution, obtained by repeating performing (i) - (iii) above, is designated vp. The intermediate value for the distance of all data in the data set $\mathrm{S}$ from the vp chosen as the root node is $\mu$. When $\mathrm{d}(\mathrm{p}, \mathrm{q})$ is established as the distance between points $\mathrm{p}, \mathrm{q}$, data set $\mathrm{S}$ is partitioned into S1 and S2 as follows: $\mathrm{S} 1=\{\mathrm{s} \in \mathrm{S} \mid \mathrm{d}(\mathrm{s}, \mathrm{vq})<\mu\} \mathrm{S} 2$ $=\{\mathrm{s} \in \mathrm{S} \mid \mathrm{d}(\mathrm{s}, \mathrm{vq}) \geq \mu\}$ (1) In like manner, this partitioning operation is recursively applied to $\mathrm{S} 1$ and $\mathrm{S} 2$ to create the index. The VP-tree index is represented by a tree structure, and subsets such as the abovementioned S1 and S2 each correspond to one node of the tree. In addition, each leaf node stores a number of data points. The search starts from the route nodes and follows the nodes conforming to the search scope, accesses data stored in the leaf node that it finally arrives at point by point, calculates the distance, and determines whether or not it conforms to the search scope.

\section{Problems with multidimensional indexing technology} in high dimensions: Content searches of images and other multimedia content employ multidimensional feature vectors that may exceed 100 dimensions. Phenomena of the kind that cannot even be imaged in two-dimensional or three-dimensional space are known to occur in such high-dimensional space. Because the degree of spatial freedom is extremely high in higherdimensional space, solving various problems in computational geometry and multivariate analysis involves an enormous amount of calculation and is hence notoriously difficult. These difficulties are collectively referred to as the "curse of dimensionality." In nearest neighbor searches in high-dimensional space, a phenomenon occurs whereby the search becomes more and more difficult as the dimensionality becomes higher. For example, when points are uniformly distributed in $\mathrm{n}$-dimensional space, the ratio of the distance of the $\mathrm{k}$-th nearest and the $(\mathrm{k}+1)$-th nearest point to a given point can be approximated by the following formula:17 $\mathrm{E}\{\mathrm{d}(\mathrm{k}+1) \mathrm{NN}\} \mathrm{E}\{\mathrm{dkNN}\} \approx 1+$ $1 \mathrm{kn}$ (2) As you can see from the above, as $\mathrm{n}$ becomes larger, the ratio of the distance of the k-th nearest point and the $(\mathrm{k}+1)$-th nearest point asymptotically approaches 1 . Moreover, when the points are uniformly distributed, the ratio of the distance to the nearest point to the distance to the most distant point asymptotically approaches 1 as the dimensionality becomes higher. Therefore, methods for dividing the space hierarchically entail problems in that the difference due to distance is small, making it impossible to limit the area explored, and an amount of calculation that approaches that of a linear search is required. 


\section{PROPOSED SCHEMA:}

A spatial info manages dimensional objects (such as points, rectangles, etc.), and provides quick access to those objects supported totally different choice criteria. The importance of spatial databases is mirrored by the convenience of modelling entities of reality in an exceedingly geometric manner. for instance, locations of restaurants, hotels, hospitals so on square measure typically described as points in an exceedingly map, whereas larger extents like parks, lakes, and landscapes typically as a mix of rectangles. several functionalities of a spatial info square measure helpful in varied ways in which in specific contexts. as an example, in an exceedingly geographics system, vary search will be deployed to search out all restaurants in an exceedingly sure space, whereas nearest neighbor retrieval will discover the eating place nearest to a given address. Furthermore, because the SI-index relies on the traditional technology of inverted index, it's without delay incorporable in an exceedingly business computer programme that applies large similarity, implying its immediate industrial deserves.

\section{NEAREST NEIGHBOR SEARCH TECHNIQUE:}

\section{A. IR-Tree, Approximation algorithm and exact algorithm:}

This method is used to retrieve a group of spatial web objects such that the query's keywords are cover by group's keywords and objects are near to the query location and have the lowest inter object distances. This method addresses the two instantiation of the group keyword query. First is to find the group of objects that cover the keywords such that the sum of their distances to the query is minimized. Second is to find a group of objects that cover the keywords such that sum of the maximum distance among an object in group of objects and query and maximum distance among two objects in group of objects is minimized. Both of these sub problems are NP-complete. Greedy algorithm is used to provide an approximation solution to the problem that utilizes the spatial keyword index IR-tree to reduce the search space. But in some application query does not contain a large number of keywords, for this exact algorithm is used that uses the dynamic programming. [1]

\section{B. IUR-tree (Intersection union R-tree)}

Geographic objects associated with descriptive texts are becoming common. This gives importance to spatial keyword queries that take both the location and text description of content. This technique is used to analyze the problem of reverse spatial and textual $\mathrm{k}$ nearest neighbor search i.e finding objects that takes the query object as one of their spatial textual similar objects. For this type of search hybrid index structure is used that successfully merge the location proximity with textual similarity. For searching, branch and bound algorithm is used. In addition to increase the speed of query processing a variant of IURtree and two optimization algorithm is used. To enhance the IURtree text clustering is used, in this objects of all the data base is group into clusters according to their text similarity. Each node of the tree is extended by the cluster information to create a hybrid tree which is called as cluster IUR-tree. To enhance the search performance of this tree two optimization methods is used, first is based on outlier detection and extraction and second method is based on text entropy. [2]

\section{C. $B R^{*}$-tree :}

This hybrid index structure is used to search m-closest keywords. This technique finds the closest tuples that matches the keywords provided by the user. This structure combines the $\mathrm{R}^{*}$-tree and bitmap indexing to process the mclosest keyword query that returns the spatially closest objects matching $\mathrm{m}$ keywords To reduce the search space a priori based search strategy is used. Two monotone constraints is used as a priori properties to facilitates efficient pruning which is called as distance mutex and keyword mutex. But this approach is not suitable for handling ranking queries and in this number of false hits is large.[3]

\section{IR2 -tree :}

The growing number of applications requires the efficient execution of nearest neighbor queries which is constrained by the properties of spatial objects. Keyword search is very popular on the internet so these applications allow users to give list of keywords that spatial objects should contain. Such queries called as a spatial keyword query. This is consisted of query area and set of keywords. The IR2 -tree is developed by the combination of R-tree and signature files, where each node of tree has spatial and keyword information. This method is efficiently answering the top-k spatial keyword queries. In this signature is added to the every node of the tree. An able algorithm is used to answer the queries using the tree. Incremental nearest algorithm is used for the tree traversal and if root node signature does not match the query signature then it prunes the whole subtrees. But IR2 -tree has some drawbacks such as false hits where the object of final 
result is far away from the query or this is not suitable for handling ranking queries.[4]

\section{E. Spatial inverted index and Minimum bounding method:}

So, new access method spatial inverted access method is used to remove the drawbacks of previous methods such as false hits. This method is the variant of inverted index using for multidimensional points. This index stores the spatial region of data points and on every inverted list Rtree is built. Minimum bounding method is used for traversing the tree to prune the search space.

\section{Processing Spatial-Keyword (SK) Queries in Geographic Information Retrieval (GIR) Systems:}

Location based information stored in GIS database. These information entities of such databases have both spatial and textual descriptions. This paper proposes a framework for GIR system and focus on indexing strategies that can process spatial keyword query. The following contributions in this paper: 1) It gives framework for query processing in Geo- graphic Information Retrieval (GIR) Systems. 2) Develop a novel indexing structure called $\mathrm{KR}^{*}$-tree that captures the joint distribution of keywords in space and significantly improves performance over existing index structures. 3) This method have conducted experiments on real GIS datasets showing the effectiveness of our techniques compared to the existing solutions. It introduces two index structures to store spatial and textual information.

\section{A) Separate index for spatial and text attributes: \\ Advantages: -}

1. Easy of maintaining two separate indices.

2. Performance bottleneck lies in the number of candidate object generated during the filtering stage.

Disadvantages: -

1. If spatial filtering is done first, many objects may lie within a query is spatial extent, but very few of them are relevant to query keywords. This increases the disk access cost by generating a large number of candidate objects. The subsequent stage of keyword filtering becomes expensive.

\section{B) Hybrid index}

Advantages and limitations: -

1. When query contains keywords that closely correlated in space, this approach suffer from paying extra disk cost accessing $\mathrm{R}^{*}$-tree and high overhead in subsequent merging process.

\section{CONCLUSION}

In this paper, we proposed a new technique for efficient nearest neighbor search in a set of high-dimensional points. Our technique is based on the pre-computation of the solution space of any arbitrary nearest-neighbor search. This corresponds to the computation of the voronoi cells of the data points. Since voronoi cells may become rather complex when going to higher dimensions, we presented a new algorithm for the approximation of high-dimensional voronoi cells using a set of minimum bounding (hyper-) rectangles. Although our technique is based on a pre-computation of the solution space, it is dynamic, i.e. it supports insertions of new data points. We finally showed in an experimental evaluation that our technique is efficient for various kinds of data and clearly outperforms the state of the art nearest-neighbor algorithms. We have seen plenty of applications calling for a search engine that is able to efficiently support novel forms of spatial queries that are integrated with keyword search. The existing solutions to such queries either incur prohibitive space consumption or are unable to give real time answers. In this paper, we have remedied the situation by developing an access method called the spatial inverted index (SI-index). Not only that the SIindex is fairly space economical, but also it has the ability to perform keyword-augmented nearest neighbor search in time that is at the order of dozens of milliseconds. Furthermore, as the SI-index is based on the conventional technology of inverted index, it is readily incorporable in a commercial search engine that applies massive parallelism, implying its immediate industrial merits.

\section{REFERENCES:}

1. S. Agrawal S. Chaudhuri, and G. Das. Dbxplorer, "A System for Keyword-based Search over Relational Databases", Proc. Of International Conference on Data Engineering (ICDE), (2002), pp. 5-16.

2. N. Beckmann, H. Kriegel, R. Schneider, and B. Seeger, "The $\mathrm{R}^{*}$-tree: An Efficient and Robust Access Method for Points and Rectangles", Proc. of ACM Management of Data (SIGMOD), (1990), pp. 322-331.

3. G. Bhalotia, A. Hulgeri, C. Nakhe, S. Chakrabarti, and S. Sudarshan, "Keyword Searching and Browsing in Databases Using Banks", Proc. of International Conference on Data Engineering (ICDE), (2002), pp. 431-440. 
4. X. Cao, L. Chen, G. Cong, C. S. Jensen, Q. Qu, A. Skovsgaard, D. Wu, and M. L. Yiu, "Spatial Keyword Querying. ER, (2012), pp. 16-29. X. Cao, G. Cong, and C. S. Jensen, "Retrieving top-k prestige-based relevant spatial web objects", PVLDB, vol. 3, no. 1, (2010), pp. 373-384.

5. X. Cao, G. Cong, C. S. Jensen, and B. C. Ooi, "Collective Spatial Keyword Querying", Proc. of ACM Management of Data (SIGMOD), (2011), pp. 373- 384 .

6. B. Chazelle, J. Kilian, R. Rubinfeld, and A. Tal, "The Bloomier Filter: An Efficient Data Structure for Static Support Lookup Tables", Proc. of the Annual ACM-SIAM Symposium on Discrete Algorithms (SODA), (2004), pp. 30-39.

7. Y.-Y. Chen, T. Suel, and A. Markowetz, "Efficient Query Processing in Geographic Web Search Engines", Proc. of ACM Management of Data (SIGMOD), (2006), pp. 277-288.

8. C. Faloutsos and S. Christodoulakis, "Signature Files: An Access Method for Documents and Its Analytical Performance Evaluation," ACM Trans. Information Systems, vol. 2, no. 4, pp. 267-288, 1984.

9. G. R. Hjaltason and H. Samet. Distance browsing in spatial databases. ACM Transactions on Database Systems (TODS), 24(2):265-318, 1999. 